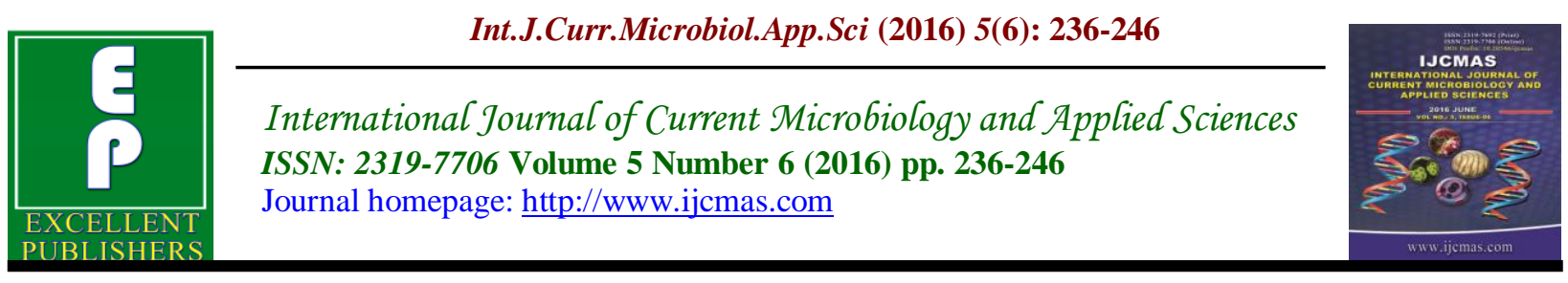

Original Research Article

http://dx.doi.org/10.20546/ijcmas.2016.506.027

\title{
Assessment of Bacterial Load in the Fresh Water Lake System of Tamil Nadu
}

\author{
R. Sugumar* and B. Anandharaj \\ Research Department of Microbiology, M. R. Government Arts College, \\ Mannargudi-614 001, Tamilnadu, India \\ *Corresponding author
}

Keywords

Freshwater,

Lakes,

Coliform Bacteria,

Bacterial

Diversity.

\begin{tabular}{l}
\hline Article Info \\
\hline Accepted: \\
12 May 2016 \\
Available Online: \\
10 June 2016
\end{tabular}

\section{A B S T R A C T}

Microbial study was done on two freshwater systems located in Viluppuram District of Tamil Nadu. Total bacterial density ranged from $6.2-14.4 \times 10^{7}$ cfu/lit in Mukaiyur lake and from $5.4-8.6 \times 10^{6} \mathrm{cfu} / \mathrm{lit}$ in Tirukoilur lake. Maximum Total Bacterial Density was noticed in November in both systems. Total coliform ranged from 165 - 1635/100 $\mathrm{ml}$ in Mugaiyur lake and from 315 - 521/100 ml in Thirukoilur lake. Mukaiyur lake recorded 22 bacterial species belonging to 16 genera, while Thirukoilur lake recorded 28 species belonging to 18 genera. In both the systems there were 11 bacterial perennial species occurred during study period.

\section{Introduction}

Microorganisms are characterized by tremendous metabolic versatility. Many important ecosystem processes rely entirely on microbial activities (e.g. nitrogen fixation). Moreover, viruses can cause bacterial diversification through gene transfers 'Weinbauer and Rassoulzadegan 2004' which in turn, has the potential to influence ecosystem functioning and the services mediated by microorganisms.

Eventhough great strides have been made in describing microbial diversity by means of modern analytical methods and a range of essential microbial-mediated processes has also been measured. Most assessments of microbial community structure and the activities that impact ecosystem functioning have been examined outside the conceptual framework of ecology and, with few exceptions, relationships between microbial biodiversity and ecosystem functioning have not been explicitly addressed.

Given the many competitive and facilitative interactions known among bacteria, changes in their biodiversity potentially have enormous scope to affect ecosystem processes (Heard and Richardson, 1995). Hence the present study was attempted to analyse the bacterial diversity in a freshwater system located in Villuppuram District, Tamil Nadu. 


\section{Materials and Methods}

The freshwater systems chosen for the present study are Mugaiyur lake and Tirukoilur lake located in Villupuram District of Tamil Nadu (Plate 1). Of these one is located in Mugaiyur village and referred to as Mugaiyur lake (Plate 2). The second lake is located in Tirukoilur town and referred to as Tirukoilur lake (Plate 3). Both the lakes are located at an elevation of about 85' MSL. The distance between two lakes is about $16 \mathrm{~km}$ and hence the general wealth conditions are almost similar to both lakes. However, both the lakes depend on varied water sources even though both are perennial. While Mugaiyur lake is rainfed, the Tirukoilur lake is purely river fed (Penniyar river of Villupuram District).

The water samples were collected by using aseptically broad mouthed sterilized glass containers, transferred to ice boxes and immediately brought to the laboratory for culturing within 6 hours. In addition, water samples were also collected in sterilized bottles for bacterial studies and transferred immediately to the laboratory in compact thermo- cold ice boxes with minimum exposure to light, temperature and undue shaking for further analyses. Water samples for both the systems were collected between 7:30 and 8:30 am.

WHO (1985) guidelines were followed for the frequency of sampling. Total bacterial count (TBC) was estimated by standard plate count using nutrient agar, while Most Probable Number (MPN), Total Coliform (TC) and faecal Streptococci (FS) were carried out by multiple tube method using Mc Conkey's and sodium azide broth respectively as recommended by ISI (1982), WHO (1985) and APHA (2000). Other biochemical tests were also done for isolation and identification of different bacterial species by using selected media and tests like IMVIC, Gram stain, fermentation, nitrate reductase, oxidase, citrate, catalase and $\mathrm{H}_{2} \mathrm{~S}$, as recommended by Jacobs and Gerstein (1960), Sirockin and Cullimore (1969), Crueckshank et al. (1975) and APHA (1985).

\section{Fluctuations of Microbiological Variables}

\section{Bacterial Abundance and Diversity (Tables 1-4)}

\section{Total Bacterial Density (TBD)}

The Total Bacterial Density in the surface water of Mugaiyur lake ranged from 6.2 to $14.4 \times 10^{7} \mathrm{cfu} / \mathrm{l}$ and for the Tirukoilur lake from 5.4 to $8.6 \times 10^{6} \mathrm{cfu} / \mathrm{l}$. For the bottom water, Mugaiyur lake recorded levels ranging from 6.4 to $14.8 \times 107 \mathrm{cfu} / \mathrm{l}$ and for the Tirukoilur lake from 6.0 to $8.8 \times 10^{7}$ cfu/l. The bottom water TBD of both systems followed the same pattern as that of surface water recording minimum levels in January and maximum in November. However a comparison of TBD levels reveals that bottom water recorded higher levels than surface water in both the systems (Tables 1 and 2). Similar observations were also noticed by Shimna (2012), Sankar Rao (2013) and Mugilan (2014). The maximum TBD noticed during November in present study is due to the rains resulting in the mixing of waters along with run-off water entering the systems bringing nutrients for enabling the growth of the organisms while the minimal values noticed in January may be due to the lower water temperature which is unsuitable for the growth of bacteria. Similar observations were also recorded by Shimna (2012), Sankarrao (2013) and Mugilan (2014). A perusal of the concentrations of Oxidable Organic Mater, Ammonia nitrogen, Nitrate-nitrogen and Phosphate (OOM, $\mathrm{NH}_{3}-\mathrm{N}, \mathrm{NO}_{3}-\mathrm{N}$ and $\mathrm{PO}_{4}$ ) 
levels in the waters of both the systems reveals a higher concentration during the rainy season which could also be a reason for the highest TBC shown during this season. In the present study, correlation between TBC and oxidizable organic matter, suspended solids and ammonia showed a positive relationship indicating their effect on bacteria. The differences in bacterial count noticed during the different seasons of the year can also be attributed to the differences in temperature and suspended solid levels as suggested by many workers (Seki, 1972; Fukami et al., 1983; Sivakami et al., 2011, 2012) in addition to oxidizable organic matter and nutrients (Sivakami et al., 2012; Sankarrao, 2013).

A comparison of the nutrient levels in different seasons also reveal that the TBC was high when the nutrient levels were also high. Correlation between TBC and nutrients like phosphates, nitrates and sulphates also a revealed positive correlation in both the systems. However, Jones (1971), suggested that temperature, $\mathrm{pH}$ and oxygen are the main limiting factors controlling the vertical bacterial population. A comparison of TBC among the two systems reveals that Mugaiyur lake recorded higher levels than Tirukoilur lake. This is probably due to higher amount of suspended solids, oxidizable organic matter as well as nutrients in this system.

\section{Total Coliform Count}

Total Coliform Count (TCC) in the surface water of Mugaiyur lake was found to range from 165 to 1635 per $100 \mathrm{ml}$ and from 315 to 521 per $100 \mathrm{ml}$ in the Tirukoilur lake. The bottom water TCC levels in Mugaiyur lake ranged from 205 to 1685 per $100 \mathrm{ml}$ and from 330 to 585 per $100 \mathrm{ml}$ in Tirukoilur lake. Both the surface and bottom waters in both the systems followed the same pattern by recording minimal levels in January and the maximum in November (Tables $1 \& 2$ ).

In the present study, the maximum TCC that was recorded in November in both systems can again be attributed to entry of water into the system due to rains resulting in surface run-off entering the systems resulting in suspended solids, OOM and nutrients. Correlating TCC with suspended solids, $\mathrm{OOM}$ and nutrients $\left(\mathrm{NO}_{3}, \mathrm{NH}_{3}, \mathrm{SO}_{4}, \mathrm{PO}_{4}\right)$ reveals a positive correlation in both the systems. The higher TCC levels noticed in the bottom waters when compared to surface waters can again be attributed to increased amount of Suspended Solids and Oxidable Organic Matter (SS and OOM) and nutrients in the bottom water when compared to the surface water.

Among the two systems, Mugaiyur lake recorded a higher level when compared to that of the Tirukoilur lake which may be due to higher amount of suspended solids, temperature, $\mathrm{OOM}$ and nutrients like $\mathrm{PO}_{4}$, $\mathrm{NO}_{3}$ and $\mathrm{NH}_{3}$. Literature reveals that similar findings were also suggested by Kumar and Shaka (2009), Petter et al. (2012), Shimna (2012), Sankar Rao (2013) and Mugilan (2014).

\section{Faecal Streptococci (FS)}

Faecal Streptococci in the surface water of Mugaiyur lake was found to range from 82 to 482 per $100 \mathrm{ml}$ and from 102 to 252 per $100 \mathrm{ml}$ in the Tirukoilur lake. The bottom water Faecal Streptococci count in Mugaiyur lake was found to range from 92 to 562 per $100 \mathrm{ml}$ and from 132 to 257 per $100 \mathrm{ml}$ in Tirukoilur lake. The bottom water Faecal Streptococci count followed the same pattern of surface waters in both the systems recording minimum level in January and maximum in November (Tables 1 and 2). 
Table.1 Bacteriological Studies in Mugaiyur Lake Water

\begin{tabular}{|c|c|c|c|c|c|c|c|c|c|c|c|c|}
\hline \multirow[b]{3}{*}{ Month } & \multicolumn{4}{|c|}{$\begin{array}{c}\text { Total Bacterial Density (TBD) } \\
\text { (per } 100 \mathrm{ml})\end{array}$} & \multicolumn{4}{|c|}{$\begin{array}{c}\text { Total Coliforms Count (TCC) } \\
\text { (per } 100 \mathrm{ml})\end{array}$} & \multicolumn{4}{|c|}{$\begin{array}{c}\text { Faecal Streptococci Count (FSC) } \\
\text { (per } 100 \mathrm{ml})\end{array}$} \\
\hline & \multicolumn{2}{|c|}{ Surface water } & \multicolumn{2}{|c|}{ Bottom water } & \multicolumn{2}{|c|}{ Surface water } & \multicolumn{2}{|c|}{ Bottom water } & \multicolumn{2}{|c|}{ Surface water } & \multicolumn{2}{|c|}{ Bottom water } \\
\hline & 2013 & 2014 & 2013 & 2014 & 2013 & 2014 & 2013 & 2014 & 2013 & 2014 & 2013 & 2014 \\
\hline January & 6.2 & 6.7 & 6.4 & 6.9 & 165 & 225 & 205 & 295 & 112 & 82 & 142 & 92 \\
\hline February & 6.7 & 6.9 & 6.7 & 7.0 & 205 & 235 & 335 & 315 & 142 & 102 & 152 & 122 \\
\hline March & 7.6 & 7.6 & 7.8 & 7.8 & 305 & 345 & 385 & 375 & 162 & 142 & 172 & 161 \\
\hline April & 7.8 & 7.8 & 7.9 & 8.0 & 345 & 375 & 410 & 405 & 170 & 151 & 182 & 182 \\
\hline May & 8.1 & 8.2 & 8.0 & 8.4 & 375 & 435 & 485 & 445 & 172 & 162 & 192 & 192 \\
\hline June & 8.4 & 8.7 & 8.2 & 8.9 & 390 & 485 & 505 & 515 & 177 & 172 & 205 & 192 \\
\hline July & 9.1 & 8.8 & 8.4 & 9.0 & 410 & 510 & 555 & 585 & 181 & 182 & 220 & 212 \\
\hline August & 10.6 & 9.0 & 10.8 & 9.1 & 465 & 535 & 605 & 665 & 184 & 197 & 246 & 231 \\
\hline September & 10.8 & 9.2 & 11.0 & 9.8 & 605 & 585 & 635 & 735 & 242 & 242 & 262 & 252 \\
\hline October & 13.2 & 12.9 & 13.4 & 13.2 & 805 & 735 & 825 & 985 & 312 & 302 & 352 & 332 \\
\hline November & 14.4 & 13.6 & 14.8 & 13.9 & 1445 & 1635 & 1805 & 1685 & 482 & 442 & 562 & 562 \\
\hline December & 11.4 & 11.2 & 11.9 & 11.8 & 865 & 945 & 925 & 1035 & 362 & 352 & 402 & 392 \\
\hline
\end{tabular}


Table.2 Bacteriological Studies in Tirukoilur Lake

\begin{tabular}{|c|c|c|c|c|c|c|c|c|c|c|c|c|}
\hline \multirow[b]{3}{*}{ Month } & \multicolumn{4}{|c|}{$\begin{array}{c}\text { Total Bacterial Density (TBD) } \\
\text { (per } 100 \mathrm{ml})\end{array}$} & \multicolumn{4}{|c|}{$\begin{array}{c}\text { Total Coliforms Count (TCC) } \\
\text { (per } 100 \mathrm{ml})\end{array}$} & \multicolumn{4}{|c|}{$\begin{array}{c}\text { Faecal Streptococci Count (FSC) } \\
\text { (per } 100 \mathrm{ml})\end{array}$} \\
\hline & \multicolumn{2}{|c|}{ Surface water } & \multicolumn{2}{|c|}{ Bottom water } & \multicolumn{2}{|c|}{ Surface water } & \multicolumn{2}{|c|}{ Bottom water } & \multicolumn{2}{|c|}{ Surface water } & \multicolumn{2}{|c|}{ Bottom water } \\
\hline & 2013 & 2014 & 2013 & 2014 & 2013 & 2014 & 2013 & 2014 & 2013 & 2014 & 2013 & 2014 \\
\hline January & 5.4 & 6.0 & 6.0 & 6.6 & 315 & 325 & 330 & 336 & 102 & 109 & 122 & 141 \\
\hline February & 5.8 & 6.2 & 6.2 & 6.8 & 345 & 341 & 350 & 371 & 107 & 117 & 127 & 132 \\
\hline March & 5.9 & 6.8 & 6.4 & 7.0 & 360 & 365 & 366 & 375 & 109 & 119 & 132 & 137 \\
\hline April & 6.1 & 6.9 & 6.5 & 7.2 & 385 & 381 & 390 & 396 & 112 & 123 & 134 & 147 \\
\hline May & 6.4 & 7.1 & 6.7 & 7.4 & 390 & 396 & 401 & 405 & 123 & 128 & 139 & 152 \\
\hline June & 6.7 & 7.3 & 7.1 & 7.5 & 410 & 401 & 422 & 411 & 142 & 132 & 147 & 157 \\
\hline July & 6.8 & 7.5 & 7.4 & 7.7 & 415 & 422 & 430 & 455 & 172 & 155 & 177 & 187 \\
\hline August & 7.0 & 7.6 & 8.0 & 7.9 & 445 & 449 & 460 & 471 & 192 & 162 & 197 & 192 \\
\hline September & 7.1 & 7.8 & 8.1 & 8.2 & 465 & 482 & 490 & 495 & 202 & 192 & 217 & 212 \\
\hline October & 7.4 & 8.1 & 8.3 & 8.4 & 475 & 498 & 516 & 524 & 212 & 222 & 227 & 237 \\
\hline November & 8.6 & 8.2 & 8.8 & 8.5 & 490 & 521 & 584 & 585 & 232 & 252 & 247 & 257 \\
\hline December & 7.2 & 7.8 & 7.4 & 7.5 & 415 & 435 & 425 & 465 & 176 & 182 & 217 & 207 \\
\hline
\end{tabular}


Table.3 Seasonal Changes in Bacterial Composition of Mugaiyur Lake

\begin{tabular}{|c|c|c|c|c|c|c|c|c|c|c|c|c|c|c|}
\hline No. & Species & Year & Jan & Feb & Mar & Apr & May & Jun & Jul & Aug & Sep & Oct & Nov & Dec \\
\hline \multirow[t]{2}{*}{1.} & \multirow[t]{2}{*}{ Aerobacter aerogenes } & 2013 & + & + & + & + & + & + & + & + & + & ++ & +++ & +++ \\
\hline & & 2014 & + & + & + & + & + & + & + & + & + & ++ & +++ & +++ \\
\hline \multirow[t]{2}{*}{2.} & \multirow[t]{2}{*}{ Aeromonas hydrophila } & 2013 & + & + & + & + & + & + & + & + & + & ++ & ++ & ++ \\
\hline & & 2014 & + & + & + & + & + & + & + & + & + & ++ & ++ & ++ \\
\hline \multirow[t]{2}{*}{3.} & \multirow[t]{2}{*}{ Bacillus cereus } & 2013 & + & + & + & - & - & - & + & + & + & ++ & ++ & ++ \\
\hline & & 2014 & + & + & + & - & - & - & + & + & + & ++ & ++ & ++ \\
\hline \multirow[t]{2}{*}{4.} & \multirow[t]{2}{*}{ Bacillus firmus } & 2013 & ++ & ++ & ++ & ++ & ++ & ++ & ++ & ++ & +++ & +++ & +++ & +++ \\
\hline & & 2014 & ++ & + & ++ & ++ & +++ & ++ & ++ & ++ & ++ & +++ & +++ & ++++ \\
\hline \multirow[t]{2}{*}{5.} & \multirow{2}{*}{ Bacillus subtilis } & 2013 & +++ & +++ & +++ & +++ & ++ & ++ & ++ & ++ & +++ & ++++ & ++++ & ++++ \\
\hline & & 2014 & +++ & +++ & ++ & +++ & +++ & ++ & + & ++ & ++ & ++++ & ++++ & ++++ \\
\hline \multirow[t]{2}{*}{6.} & \multirow[t]{2}{*}{ Clostridium perfringens } & 2013 & + & - & - & - & - & + & + & + & + & + & ++ & ++ \\
\hline & & 2014 & + & - & - & - & - & - & + & + & + & + & ++ & ++ \\
\hline \multirow[t]{2}{*}{7.} & \multirow[t]{2}{*}{ Enterobacter aerogenes } & 2013 & + & + & + & + & + & + & + & + & ++ & ++ & ++ & + \\
\hline & & 2014 & + & + & + & + & + & + & + & + & ++ & ++ & ++ & + \\
\hline \multirow[t]{2}{*}{8.} & \multirow[t]{2}{*}{ Enterobacter cloacae } & 2013 & + & + & + & - & - & - & - & + & + & + & + & + \\
\hline & & 2014 & + & + & + & - & - & - & - & - & + & + & + & + \\
\hline \multirow[t]{2}{*}{9.} & \multirow[t]{2}{*}{ Erwinia rhapontici } & 2013 & + & - & - & - & - & - & - & - & - & + & + & + \\
\hline & & 2014 & - & - & - & - & - & - & - & - & - & - & - & - \\
\hline \multirow[t]{2}{*}{10.} & \multirow[t]{2}{*}{ Escherichia coli } & 2013 & ++++ & ++++ & +++ & +++ & ++++ & +++ & +++ & +++ & ++++ & ++++ & +++++ & +++++ \\
\hline & & 2014 & ++++ & ++++ & +++ & +++ & ++++ & +++ & +++ & +++ & ++++ & ++++ & ++++ & ++++ \\
\hline \multirow[t]{2}{*}{11.} & \multirow[t]{2}{*}{ Flavobacterium johnsoniae } & 2013 & +++ & +++ & +++ & +++ & +++ & ++ & ++ & ++ & +++ & +++ & +++ & +++ \\
\hline & & 2014 & +++ & ++ & ++ & ++ & ++ & ++ & ++ & +++ & ++ & +++ & +++ & +++ \\
\hline \multirow[t]{2}{*}{12.} & \multirow[t]{2}{*}{ Klebsiella pneumoniae } & 2013 & + & + & + & - & - & + & + & + & ++ & ++ & ++ & + \\
\hline & & 2014 & + & + & + & - & - & + & + & + & ++ & ++ & ++ & ++ \\
\hline \multirow[t]{2}{*}{13.} & \multirow[t]{2}{*}{ Proteus mirabilis } & 2013 & +++ & +++ & + & ++ & ++ & ++ & ++ & ++ & ++ & ++ & +++ & +++ \\
\hline & & 2014 & ++ & ++ & + & + & + & + & + & + & + & + & +++ & +++ \\
\hline \multirow[t]{2}{*}{14.} & Proteus vulgaris & 2013 & + & + & + & + & + & - & - & + & + & + & +++ & +++ \\
\hline & & 2014 & + & + & + & + & + & - & - & - & - & + & +++ & +++ \\
\hline
\end{tabular}


Table-3 continued....

\begin{tabular}{|c|c|c|c|c|c|c|c|c|c|c|c|c|c|c|}
\hline No. & Species & Year & Jan & Feb & Mar & Apr & May & Jun & Jul & Aug & Sep & Oct & Nov & Dec \\
\hline \multirow[t]{2}{*}{15.} & \multirow[t]{2}{*}{ Pseudomonas aeruginosa } & 2013 & +++ & +++ & ++ & ++ & ++ & ++ & ++ & ++ & +++ & ++++ & ++++ & ++++ \\
\hline & & 2014 & +++ & +++ & ++ & ++ & ++ & ++ & ++ & ++ & +++ & ++++ & ++++ & ++++ \\
\hline \multirow[t]{2}{*}{16.} & \multirow[t]{2}{*}{ Salmonella typhi } & 2013 & + & - & - & - & - & - & + & + & + & + & + & + \\
\hline & & 2014 & + & - & - & - & - & - & - & + & + & + & + & + \\
\hline \multirow[t]{2}{*}{17.} & \multirow[t]{2}{*}{ Serratia marcescens } & 2013 & + & + & + & + & - & - & - & - & - & + & + & + \\
\hline & & 2014 & + & + & + & + & - & - & - & - & - & + & + & + \\
\hline \multirow[t]{2}{*}{18.} & \multirow[t]{2}{*}{ Shigella sonnei } & 2013 & - & - & - & - & - & - & - & - & - & - & - & - \\
\hline & & 2014 & - & - & - & - & - & - & - & - & + & + & + & + \\
\hline \multirow[t]{2}{*}{19.} & \multirow[t]{2}{*}{ Staphylococcus aureus } & 2013 & ++ & ++ & ++ & ++ & + & ++ & ++ & +++ & +++ & +++ & ++++ & ++++ \\
\hline & & 2014 & ++ & ++ & ++ & ++ & + & ++ & ++ & +++ & +++ & +++ & +++ & ++++ \\
\hline \multirow[t]{2}{*}{20.} & \multirow[t]{2}{*}{ Streptococcus faecalis } & 2013 & + & + & + & + & + & + & + & ++ & ++ & ++ & ++ & ++ \\
\hline & & 2014 & + & + & + & + & + & + & + & ++ & ++ & ++ & ++ & ++ \\
\hline \multirow[t]{2}{*}{21.} & \multirow[t]{2}{*}{ Vibrio alginolyticus } & 2013 & - & - & - & - & - & - & - & - & - & - & - & - \\
\hline & & 2014 & + & + & - & - & - & - & - & - & - & + & + & + \\
\hline \multirow[t]{2}{*}{22.} & \multirow[t]{2}{*}{ Vibrio cholerae } & 2013 & + & + & + & - & - & - & + & + & + & + & + & + \\
\hline & & 2014 & + & + & - & - & - & - & + & + & + & + & + & + \\
\hline
\end{tabular}

'+' represents Presence

'-' represents Absence 
Table.4 Seasonal Changes in Bacterial Composition of Tirukoilur Lake

\begin{tabular}{|c|c|c|c|c|c|c|c|c|c|c|c|c|c|c|}
\hline No. & Species & Year & Jan & Feb & Mar & Apr & May & Jun & Jul & Aug & Sep & Oct & Nov & Dec \\
\hline \multirow[t]{2}{*}{1.} & \multirow[t]{2}{*}{ Aerobacter aerogenes } & 2013 & ++ & ++ & + & + & + & + & + & + & + & + & + & + \\
\hline & & 2014 & ++ & ++ & + & + & + & + & + & + & + & + & + & + \\
\hline \multirow[t]{2}{*}{2.} & \multirow[t]{2}{*}{ Aeromonas hydrophila } & 2013 & +++ & +++ & +++ & ++ & ++ & ++ & ++ & +++ & +++ & +++ & ++++ & ++++ \\
\hline & & 2014 & +++ & +++ & +++ & ++ & ++ & ++ & ++ & ++ & +++ & +++ & +++ & ++++ \\
\hline \multirow[t]{2}{*}{3.} & \multirow[t]{2}{*}{ Alcaligenes denitrificans } & 2013 & + & + & - & - & - & - & - & - & ++ & ++ & ++ & ++ \\
\hline & & 2014 & - & - & - & - & - & - & - & - & - & + & + & + \\
\hline \multirow[t]{2}{*}{4.} & \multirow[t]{2}{*}{ Bacillus cereus } & 2013 & + & + & + & + & + & + & + & + & + & + & + & + \\
\hline & & 2014 & + & + & + & + & + & + & + & + & + & + & + & + \\
\hline \multirow[t]{2}{*}{5.} & \multirow{2}{*}{ Bacillus megaterium } & 2013 & + & ++ & ++ & ++ & + & ++ & + & ++ & ++ & ++ & ++ & ++ \\
\hline & & 2014 & + & ++ & ++ & + & + & ++ & + & ++ & ++ & ++ & ++ & ++ \\
\hline \multirow[t]{2}{*}{6.} & \multirow[t]{2}{*}{ Bacillus subtilis } & 2013 & ++ & ++ & ++ & ++ & ++ & ++ & ++ & +++ & +++ & +++ & +++ & +++ \\
\hline & & 2014 & ++ & ++ & ++ & ++ & ++ & ++ & ++ & ++ & +++ & +++ & +++ & +++ \\
\hline \multirow[t]{2}{*}{7.} & \multirow[t]{2}{*}{ Chromobacterium sp. } & 2013 & + & + & - & - & - & - & - & - & - & + & + & + \\
\hline & & 2014 & + & + & - & - & - & - & - & - & - & + & + & + \\
\hline \multirow[t]{2}{*}{8.} & \multirow[t]{2}{*}{ Clostridium perfringens } & 2013 & + & + & - & - & - & - & - & + & + & + & + & + \\
\hline & & 2014 & + & + & - & - & - & - & - & + & + & + & + & + \\
\hline \multirow[t]{2}{*}{9.} & \multirow[t]{2}{*}{ Enterobacter aerogenes } & 2013 & ++ & ++ & ++ & - & - & - & - & ++ & ++ & ++ & ++ & ++ \\
\hline & & 2014 & ++ & ++ & ++ & - & - & - & - & ++ & ++ & ++ & ++ & ++ \\
\hline \multirow[t]{2}{*}{10.} & \multirow[t]{2}{*}{ Erwinia rhapontici } & 2013 & - & - & - & - & - & - & - & - & + & + & + & + \\
\hline & & 2014 & - & - & - & - & - & - & - & - & + & + & + & + \\
\hline \multirow[t]{2}{*}{11.} & \multirow[t]{2}{*}{ Escherichia coli } & 2013 & +++ & +++ & +++ & +++ & +++ & +++ & +++ & +++ & ++++ & ++++ & +++ & +++ \\
\hline & & 2014 & +++ & +++ & +++ & +++ & ++ & ++ & ++ & +++ & ++++ & ++++ & +++ & +++ \\
\hline \multirow[t]{2}{*}{12.} & \multirow[t]{2}{*}{ Flavobacterium johnsoniae } & 2013 & + & + & + & + & + & + & + & + & + & + & + & + \\
\hline & & 2014 & + & + & + & + & + & + & + & + & + & + & + & + \\
\hline \multirow[t]{2}{*}{13.} & \multirow[t]{2}{*}{ Klebsiella pneumoniae } & 2013 & + & + & + & + & + & + & + & + & + & + & + & + \\
\hline & & 2014 & + & + & + & + & + & + & + & + & + & + & + & + \\
\hline \multirow[t]{2}{*}{14.} & Proteus mirabilis & 2013 & + & + & + & - & - & - & - & - & - & + & + & + \\
\hline & & 2014 & + & + & - & - & - & - & - & - & - & + & + & + \\
\hline
\end{tabular}


Table- 4 continued....

\begin{tabular}{|c|c|c|c|c|c|c|c|c|c|c|c|c|c|c|}
\hline No. & Species & Year & Jan & Feb & Mar & Apr & May & Jun & Jul & Aug & Sep & Oct & Nov & Dec \\
\hline \multirow[t]{2}{*}{15.} & \multirow[t]{2}{*}{ Proteus vulgaris } & 2013 & + & ++ & ++ & ++ & + & ++ & ++ & +++ & +++ & +++ & +++ & +++ \\
\hline & & 2014 & + & ++ & ++ & ++ & + & ++ & ++ & +++ & +++ & +++ & +++ & +++ \\
\hline \multirow[t]{2}{*}{16.} & \multirow{2}{*}{ Pseudomonas aeruginosa } & 2013 & ++++ & ++++ & ++++ & +++ & ++ & ++ & +++ & +++ & ++++ & ++++ & ++++ & ++++ \\
\hline & & 2014 & ++++ & +++ & +++ & +++ & ++ & ++ & +++ & +++ & ++++ & ++++ & ++++ & +++++ \\
\hline \multirow[t]{2}{*}{17.} & \multirow[t]{2}{*}{ Pseudomonas agarolyticus } & 2013 & + & + & + & + & - & + & + & + & + & + & + & + \\
\hline & & 2014 & - & + & + & + & - & + & + & - & + & + & + & + \\
\hline \multirow[t]{2}{*}{18.} & \multirow{2}{*}{ Salmonella typhi } & 2013 & + & - & + & + & - & - & - & + & + & + & + & + \\
\hline & & 2014 & + & + & + & + & - & - & - & - & + & + & + & + \\
\hline \multirow[t]{2}{*}{19.} & \multirow[t]{2}{*}{ Serratia marcescens } & 2013 & - & - & - & + & + & + & - & + & + & + & - & - \\
\hline & & 2014 & + & + & + & - & - & - & - & + & + & + & + & + \\
\hline \multirow[t]{2}{*}{20.} & \multirow{2}{*}{ Shigella flexneri } & 2013 & - & - & - & - & - & - & - & - & - & - & - & - \\
\hline & & 2014 & + & + & + & + & + & - & - & - & ++ & ++ & ++ & ++ \\
\hline \multirow[t]{2}{*}{21.} & \multirow[t]{2}{*}{ Shigella sonnei } & 2013 & + & + & + & + & + & - & - & - & ++ & +++ & +++ & ++ \\
\hline & & 2014 & + & + & + & + & + & - & - & - & ++ & +++ & +++ & ++ \\
\hline \multirow[t]{2}{*}{22.} & \multirow[t]{2}{*}{ Staphylococcus aureus } & 2013 & + & + & + & + & + & + & + & + & ++ & ++ & ++ & ++ \\
\hline & & 2014 & + & + & + & + & + & + & + & + & ++ & ++ & ++ & ++ \\
\hline \multirow[t]{2}{*}{23.} & \multirow{2}{*}{ Streptococcus bovis } & 2013 & ++ & ++ & ++ & + & + & + & + & + & ++ & +++ & +++ & +++ \\
\hline & & 2014 & ++ & ++ & ++ & + & + & + & + & + & ++ & +++ & +++ & +++ \\
\hline \multirow[t]{2}{*}{24.} & \multirow{2}{*}{ Streptococcus equinus } & 2013 & + & + & + & - & - & - & - & + & + & + & ++ & ++ \\
\hline & & 2014 & + & + & + & - & - & - & - & + & + & + & ++ & ++ \\
\hline \multirow[t]{2}{*}{25.} & \multirow[t]{2}{*}{ Streptococcus faecalis } & 2013 & ++ & ++ & ++ & + & - & - & + & ++ & ++ & ++ & ++ & ++ \\
\hline & & 2014 & ++ & + & ++ & + & - & - & + & ++ & ++ & ++ & ++ & ++ \\
\hline \multirow[t]{2}{*}{26.} & \multirow[t]{2}{*}{ Vibrio alginolyticus } & 2013 & + & + & + & - & - & - & - & - & - & ++ & ++ & ++ \\
\hline & & 2014 & + & + & - & - & - & - & - & - & - & ++ & ++ & ++ \\
\hline \multirow[t]{2}{*}{27.} & \multirow[t]{2}{*}{ Vibrio cholerae } & 2013 & - & - & + & - & + & - & - & - & - & ++ & ++ & ++ \\
\hline & & 2014 & - & - & + & - & + & - & - & - & - & ++ & ++ & ++ \\
\hline \multirow[t]{2}{*}{28.} & Vibrio parahaemolyticus & 2013 & + & - & - & - & - & - & + & + & + & + & + & + \\
\hline & & 2014 & + & - & - & - & - & - & + & + & + & + & + & + \\
\hline
\end{tabular}

‘+' represents Presence; '-' represents Nil 
Vasconcelos and Swartz (1976) reported that viability of faecal and coliform bacteria is inversely proportional to temperature, since high temperature enhances the growth of such bacteria in water. In the present study also both the systems recorded the maximum counts during rainy season (November) when temperature was low. Statistical correlation of Faecal Streptococci, total coliform count and total bacterial count with temperature showed a negative correlation (Annexures 1 to 10) thus showing their interrelationship.

\section{Bacterial Species Diversity}

Tables 3 and 4 reveal in Mugaiyur lake a total of 22 species were noticed which belonged to 16 genera while in the Tirukoilur lake, a total of 28 species belonging to 18 genera were recorded with both the systems recording 11 perennial species each. Among the perennial species, seven species (E. coli, S. aureus, B. subtilis, $A$. aerogenes, $F$. johnsomae, $P$. aeruginosa and $A$. hydrophila) were common to both the systems while one species was unique (Enterobacter cloacae) to Mugaiyur lake while for Tirukoilur lake five were unique (Alcaligenes denifrificans, Bacillus megaterium, Shigella flexneri, Streptococcus bovis and $S$. equines). Further, in both the systems $E$. coli and $P$. aeruginosa were the dominant species in terms of count even though in Mugaiyur lake $F$. johnsomae also dominated while in Tirukoilur lake, $A$. hydrophila also dominated. With regard to the least dominant species. Mugaiyur lake recorded $S$. sonneri and $V$. alginolyticus while in Tirukoilur lake it was $E$. rhapontici and E. nurabilis (Tables 3 and 4).

With regard to their most favourable period of occurrence in maximal numbers, total bacterial count as a whole in both the systems recorded higher levels during the period between October and December even though each species appeared to occur in highest counts in different months of the year. Thus both the systems showed both uniqueness and similarity between them. Between the two systems, Tirukoilur lake recorded higher diversity in terms of both species as well as genera. This variation may be due to the differences in nutrient levels as reported by other workers (Lim and Flint, 1989; Bogosian et al., 1996; Shimna, 2012; Sankar Rao, 2013; Mugilan, 2014).

In the present study, the presence of Vibrio, Aeromonas, E. coli, Enterobacter, Staphylococcus species etc. indicates the presence of significant levels of microbial pollution. The presence of $E$. coli in both the systems possibly indicates faecal contamination. According to Kataria and Ambhore (2012) the presence of E. coli indicates potentially dangerous contamination requiring immediate attention. Hence this requires immediate attention if such systems are used for the betterment of man.

\section{References}

APHA. 2000. Standard methods for the examination of water and wastewater. $21^{\text {st }}$ edition. American Public Health Association, Washington, USA, p. 2240.

Bogosian, G., Sammons, L.E., Morris, P.J. L., O’Neil, J.P., Heitkamp, M.A., Weber, D.B. 1996. Death of the Escherichia coli k-12 strain w3110 in soil and water. Appl. Environ. Microbiol., 62: 4114-4120.

Cruickshank, R., Duguid, I.P., Marmion, B.P., Swain, R.A.H. 1975. In: Medical Microbiology. $12^{\text {th }}$ Ed. Churchill Livingstone, Edinburg, London.

Fukami, K., Slimidu, U., Taga, N. 1983. Distribution of heterotrophic bacteria in relation to the concentration of particulate organic matter in sea water. Can. J. Microbiol., 29: 570-575. 
Heard, S.B., Richardson, J.S. 1995. Shreddercollector facilitation in stream detrital food webs: Is there enough evidence? Oikos, 72: 359-366.

ISI. 1982. Indian Standards: Methods of sampling and microbiological examination of water. Indian Standard Institutions, New Delhi. p. 164

Jacobs, M.B., Gerstein, M.J. 1960. Handbook of Microbiology. Van Nostrand Reinhold Co., USA, p. 322.

Jones, J.G. 1971. Studies on fresh water bacteria, factors that influence the population and its activity. J. Ecol., 59: 593-613.

Kataria, H.C., Ambhore, S. 2012. Pre- and post-monsoon physico-chemical assessment of drinking water quality of Gandhinagar Area of Bhopal. Current World Environment, 7(2): 309-311.

Kumar, S., Saha, L.L. 2009. Incidence of indicator bacteria in different drinking water sources. In: Assessment of water pollution (Ed. S. R. Mishra). A. P. H. Publishing Corporation, New Delhi.

Lim, C.H., Flint, K.P. 1989. The effects of nutrients on the survival of Escherichia coli in lake water. J. Appl. Bacter., 66: 559-569.

Mugilan, V. 2014. Biodiversity of Microorganisms in Two Different Fresh Water Ecosystems and Their Role in Environmental Pollution Abatement, Ph.D. Thesis, Bharathidasan University, Tiruchirappalli, India.

Peter, L.H., Bykova, O., Dyke, J., Etienne, R.S., Hickler, T., Kuhn, I., Marion, G., Ohlemuller, R., Schymanski, S.J., Singer, A. 2012. Biotoic modifiers, environmental modulation and species distribution models. J. Ecol., 10: 1365-1369.

Vasconcelos, G.J., Swartz, R.G. 1976. Survival of bacteria in seawater using a diffusion chamber apparatus in situ. Appl. Environ. Microbiol., 31: 913-920.

Sankar Rao, M. 2013. A study on seasonal variations of microorganisms in temple ponds and its role in antimicrobial activity. Ph.D. Thesis, Bharathidasan University, Tiruchirappalli.

Seki, H. 1972. The role of microorganisms in the marine food chain with reference to organic aggregate. Mem. $1^{\text {st }}$ Ital. Idrobiol. Dott. Macro. De Marchi, 29: 245-259.

Shimna, P.P. 2012. Microbial diversity and abundance of ponds of subtropical India. Ph.D. Thesis, Bharathidasan University, Tiruchirappalli, India.

Sirockin, G., Cullimore, S. 1969. Practical Microbiology. McGraw-Hill, London. p. 159.

Sivakami, R., Sankar Rao, M. 2011. Bacterial enumeration in surface and bottom waters of two different freshwater ecosystems in Tamil Nadu. J. Curr. Sci., 16: 91-95.

Sivakami, R., Mugilan, V., Nageema, A., Amanulla Hameed, S.V.S. 2012. A comparative study on coliform bacterial density and dynamics in relation to physico-chemical characteristics of two temporary ponds. Int. J. Univ. Pharm. and Life Sci., 2: 50-56.

Vasconcelos, G.J., Swartz, R.G. 1976. Survival of bacteria in seawater using a diffusion chamber apparatus in situ. Appl. Environ. Microbiol., 31: 913-920.

WHO. 1985. Guidelines for drinking water quality. World Health Organisation, Geneva. p. 121.

\section{How to cite this article:}

Sugumar, R., and Anandharaj, B. 2016. Assessment of Bacterial Load in the Fresh Water Lake System of Tamil Nadu. Int.J.Curr.Microbiol.App.Sci. 5(6): 236-246. doi: http://dx.doi.org/10.20546/ijcmas.2016.506.027 